\title{
Triagem Familiar no Diagnóstico da Síndrome do QT Curto após Morte Súbita Cardíaca como Primeira Manifestação em Jovens Irmãos
}

\author{
Family Screening in the Diagnosis of Short QT Syndrome after Sudden Cardiac Death as First Manifestation \\ in Young Siblings
}

\author{
Guilherme Augusto Teodoro Athayde, ${ }^{10}$ Natália Quintella Sangiorgi Olivetti, ${ }^{1}$ Francisco Carlos da Costa \\ Darrieux, ${ }^{1}$ Luciana Sacilotto, ${ }^{1 \oplus}$ Gabrielle D'Arezzo Pessente, ${ }^{10}$ Maurício Ibrahim Scanavacca ${ }^{1}$ \\ Instituto do Coração do Hospital das Clínicas da Faculdade de Medicina da Universidade de São Paulo, ${ }^{1}$ São Paulo, SP - Brasil
}

\section{Introdução}

Em 2000, Gussak et al.,1 publicaram uma série de casos em que taquiarritmias atriais, síncope e morte súbita cardíaca (MSC) se associavam a um intervalo QT pronunciadamente curto, sendo os primeiros a correlacionar todos esses achados em uma síndrome. Desde então, esforços vêm sendo realizados, a fim de melhor entender o comportamento e encontrar alternativas de tratamento para a síndrome do QT curto (SQTC); no entanto, esbarram na raridade da doença, na dificuldade em estabelecer parâmetros diagnósticos e na sua complexa manifestação clínica. Este artigo relata o caso de irmãos jovens com MSC e SQTC e versa sobre as dificuldades diagnósticas e terapêuticas da síndrome.

\section{Relato de Caso}

Um indivíduo de 18 anos de idade apresentou morte súbita enquanto tinha relações sexuais. A necrópsia realizada não evidenciou uma causa mortis precisa e não havia alterações cardíacas estruturais. Após 6 meses do ocorrido, seu irmão, aos 11 anos de idade, enquanto caminhava voltando da escola, apresentou MSC revertida, sem evidência do ritmo, sendo levado à unidade de terapia intensiva (UTI) de um hospital da região, falecendo após 9 dias. A necrópsia, mais uma vez, demonstrou um coração estruturalmente normal.

Seus familiares (Figura 1) procuraram auxílio médico para investigação diagnóstica. A história clínica revelava um passado de drogadição longínquo do pai, e o filho vivo de 18 anos apresentara síncopes sem pródromos na infância, sem relação com fatores desencadeantes sugestivos de fenômenos autonômicos. A filha viva, de 20 anos de idade, relatava palpitações com segundos de duração e tinha hipotireoidismo, assim como a mãe. O pai, o filho e o avô paterno apresentavam pectus excavatum e uma envergadura

\section{Palavras-chave}

Síndrome do QT Curto; Morte Súbita Cardíaca; Cardiodesfibrilador Implantável; Teste Genético; Canalopatias Cardíacas.

Correspondência: Maurício Ibrahim Scanavacca •

Universidade de São Paulo Instituto do Coração - Dr. Enéas de Carvalho

Aguiar, 44 CEP 05403-900, São Paulo, SP - Brasil

E-mail: mauricio.scanavacca@incor.usp.br

DOI: https://doi.org/10.36660/abc.20200274 maior que a altura, todavia sem critérios para diagnóstico de síndrome de Marfan. O eletrocardiograma (ECG) do pai mostrava um QTc de 371 ms e o do filho vivo, de $323 \mathrm{~ms}$ (Figura 2), enquanto o da filha viva, QTc de $380 \mathrm{~ms}$. Os exames ecocardiograma, ECG de alta resolução (ECG-AR), angiotomografia de aorta e ressonância magnética cardíaca de todos os familiares de primeiro grau encontravam-se sem alterações. Não se observaram arritmias atriais ou ventriculares no teste ergométrico ou no Holter de 24 horas.

Foi realizado teste genético do pai por sequenciamento de nova geração (NGS), não sendo identificadas variantes patogênicas ou possivelmente patogênicas em um painel de 101 genes que se correlacionam com MSC, incluindo SQTC (KCNH2, KCNQ1, KCNJ2, CACNA1C e CACNB2) e Marfan (FBN1 e TGFBR).

Na ausência de monitor de eventos implantáveis no sistema único de saúde, optou-se por implante de cardiodesfibrilador implantável (CDI) no filho de 18 anos, devido à história de síncopes sem pródromos, associada ao histórico familiar de MSC, e ao intervalo QTc encurtado, favoráveis ao diagnóstico de SQTC. Em seguimento de 18 meses, não apresentou síncope ou terapias pelo CDI.

\section{Discussão}

A MSC em jovens com coração estruturalmente normal definido por necrópsia pode ser considerada presumivelmente arrítmica, especificamente por canalopatias cardíacas. ${ }^{2}$ As principais canalopatias incluem síndrome do QT longo, síndrome de Brugada, taquicardia ventricular polimórfica catecolaminérgica, doença progressiva da condução cardíaca e, por fim, SQTC.

A maioria desses distúrbios está associada a mutações em genes que codificam as subunidades alfaformadoras de poros do canal e suas interações com proteínas auxiliares, responsáveis pelo funcionamento dos canais de sódio, potássio e cálcio. ${ }^{2}$ Portanto, a avaliação de familiares em um contexto de duas MSC em jovens com necrópsia normal abrange análise clínica extensa dos sobreviventes, bem como testes genéticos post mortem.

O único achado relevante na investigação cínica familiar, especificamente no filho sobrevivente que apresentava síncope "desliga-liga" e no pai, foi um intervalo QT encurtado. O diagnóstico da SQTC é alvo de debate na literatura até os dias atuais. Os critérios propostos por Gollob et al., ${ }^{3}$ em 2011, foram os primeiros a ser utilizados. ${ }^{3}$ Em 2015, novos critérios foram propostos, ${ }^{4}$ sendo a SQTC 


\section{Relato de Caso}

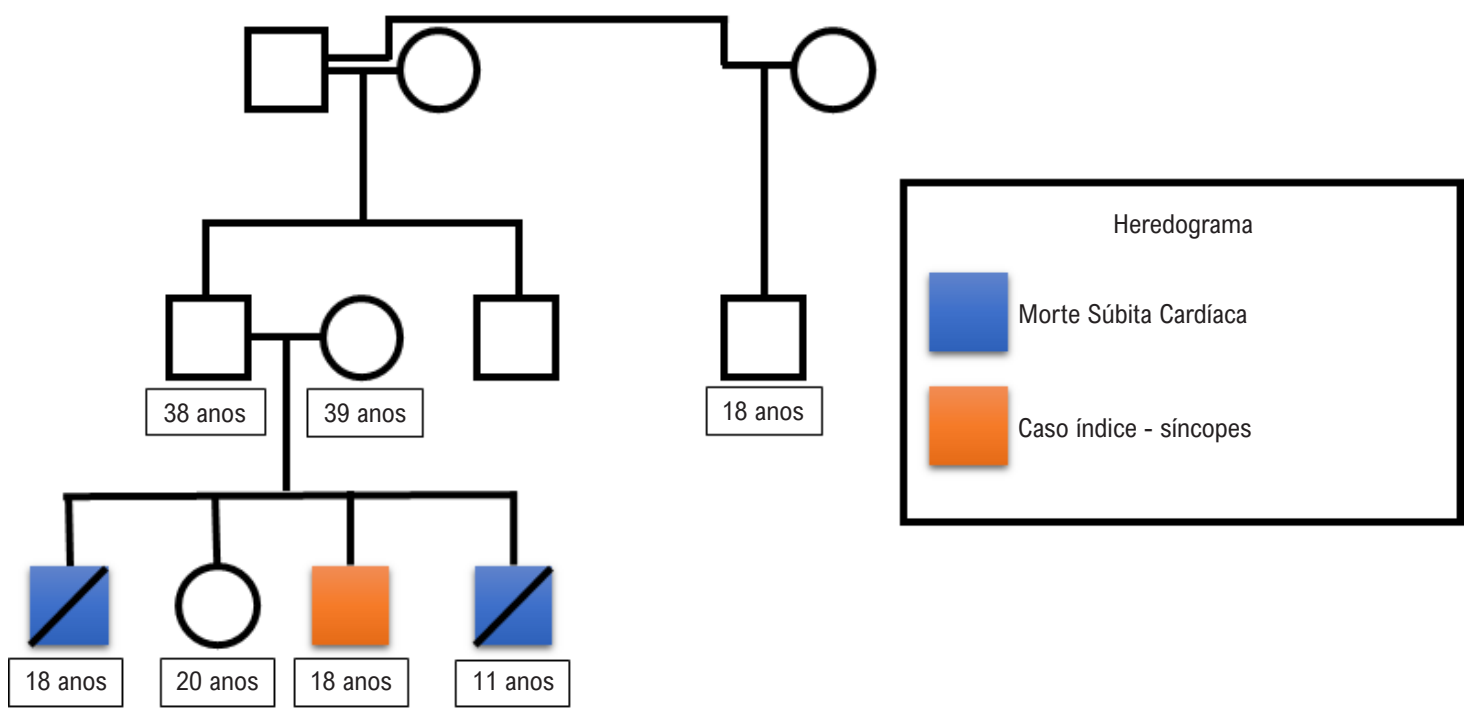

Figura 1 - Heredograma familiar, mostrando o caso-índice em laranja com manifestação de síncopes. Seus irmãos, marcados em azul, sofreram morte súbita cardiaca aos 18 anos de idade (durante atividade sexual) e aos 11 anos de idade (caminhando de volta da escola), respectivamente, da esquerda para a direita. Morte Súbita cardiaca definida conforme Priori et al: ${ }^{4}$ nenhuma causa extracardíaca óbvia ocorreu no exame post mortem e, portanto, um evento arrítmico é a causa provável da morte.

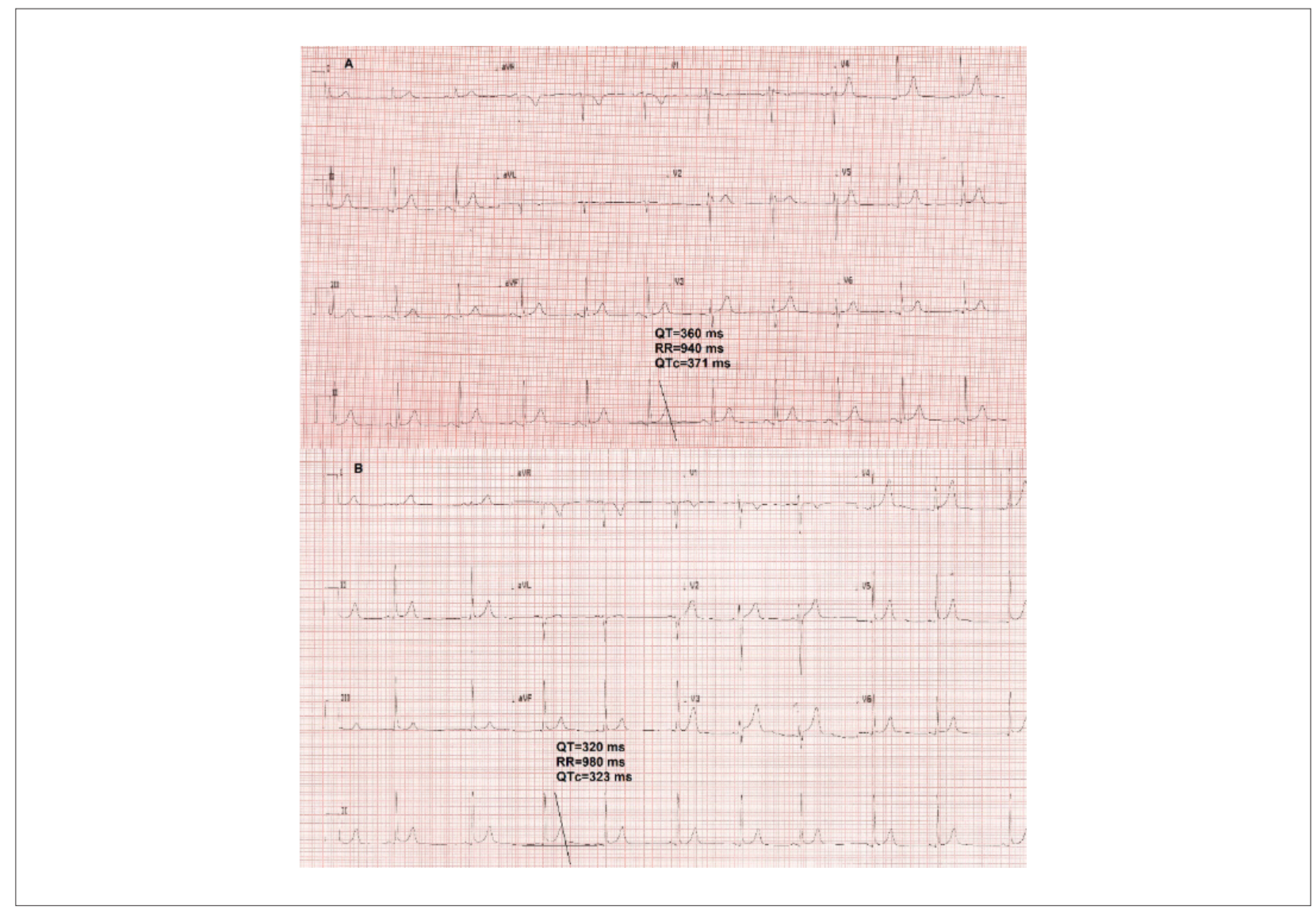

Figura 2 - Eletrocardiogramas da chegada dos pacientes ao serviço, mostrando intervalos QT curtos. A. Eletrocardiograma do pai, com QTc $=371 \mathrm{~ms}$. B. Eletrocardiograma do filho vivo, com QTc $=323 \mathrm{~ms}$. 
diagnosticada quando o intervalo QTc $\leq 340 \mathrm{~ms}$ ou quando o intervalo QTc <360 ms, em associação à história clínica, familiar ou presença de mutação patogênica. No caso exposto, o ECG do filho sobrevivente apresentava um QTc de $323 \mathrm{~ms}$, associado à forte história de MSC em familiares $\leq 40$ anos de idade, além do passado de síncopes inexplicadas. Isoladamente, um intervalo QTc $<340 \mathrm{~ms}$ é diagnóstico de SQTC e, pelo escore de Gollob, somaria 5 pontos, ${ }^{3}$ preenchendo critérios de alta probabilidade diagnóstica para a SQTC. Não documentamos fibrilação atrial até o momento, que reforçaria o diagnóstico de SQTC.

A morte súbita em irmãos jovens, sem antecedentes familiares adicionais de MSC, sugere a presença de doença recessiva, porém os pais não eram consanguíneos. Outra possibilidade é a ocorrência de mutação de novo em um dos genitores, com uma expressão clínica mais branda. O pai apresentava intervalo QT mais curto que a média populacional, porém era assintomático. Como uma doença autossômica dominante de penetrância incompleta e expressividade variável, ${ }^{5}$ ele poderia ser um carreador assintomático de uma canalopatia cardíaca expressa com maior gravidade nos filhos que faleceram. Além disso, as mortes e a síncope do sobrevivente aconteceram na segunda década de vida, mais sugestivos de TVPC, não identificada na avaliação clínica e genética. ${ }^{4}$

A real prevalência da SQTC é duvidosa em virtude de sua raridade. O encurtamento do intervalo QT reflete a repolarização acelerada, gerando uma dispersão da repolarização entre as camadas cardíacas, favorecendo o mecanismo de reentrada funcional nos átrios e ventrículos, predispondo à fibrilação atrial e ventricular. ${ }^{6}$

Em canalopatias, o teste genético tem papel limitado na síndrome de Brugada e na SQTC (cerca de 25\%), ,,7 diferentemente da SQTL $(80 \%)^{8}$ e da TVPC (90\%). ${ }^{9}$ Logo, um teste genético sem identificação de mutações patogênicas ocorre com mais frequência na $\mathrm{SQTC}^{3}$ e não exclui o diagnóstico de SQTC.

$\mathrm{O}$ CDI tem se mostrado como o tratamento mais efetivo e seguro para os pacientes com SQTC, uma vez que estes apresentam alto risco de MSC..$^{10} \mathrm{O}$ implante de CDI é recomendado para pacientes com diagnóstico de SQTC que são sobreviventes de uma MSC ou que têm taquicardia ventricular (TV) sustentada espontânea documentada. ${ }^{4}$ No nosso caso, o tratamento proposto foi o implante de CDI no filho vivo e a observação clínica do restante da família, de acordo com as recomendações mais recentes que sugerem que um CDI poderia ser considerado em pacientes com SQTC com uma forte história familiar de MSC e uma evidência de QTc curto, como classe de recomendação $\mathrm{llb}{ }^{4}$

\section{Conclusão}

O diagnóstico etiológico da MSC em jovens pode ser desafiador, principalmente quando há suspeita de morte súbita presumivelmente arrítmica, por sua característica de penetrância incompleta e expressividade variável. A síndrome do QT curto é uma canalopatia muito rara e, portanto, com uma caracterização fenotípica limitada, que deve ser recordada no cenário de MSC de etiologia desconhecida em jovens. Seu diagnóstico baseia-se apenas no ECG, na história clínica e familiar e na genética. O CDI é a principal arma terapêutica, mostrando-se um tratamento efetivo e seguro para a redução da mortalidade nesses pacientes.

\section{Contribuição dos autores}

Concepção e desenho da pesquisa e Revisão crítica do manuscrito quanto ao conteúdo intelectual importante: Athayde GAT, Olivetti NQS, Darrieux FCC, Sacilotto L, Scanavacca MI; Obtenção de dados: Athayde GAT, Olivetti NQS, Pessente GD; Análise e interpretação dos dados: Athayde GAT, Darrieux FCC, Sacilotto L, Pessente GD; Redação do manuscrito: Athayde GAT, Darrieux FCC, Sacilotto L.

\section{Potencial conflito de interesse}

Não há conflito com o presente artigo

\section{Fontes de financiamento}

O presente estudo não teve fontes de financiamento externas.

\section{Vinculação acadêmica}

Não há vinculação deste estudo a programas de pós-graduação.

\section{Aprovação ética e consentimento informado}

Este artigo não contém estudos com humanos ou animais realizados por nenhum dos autores.

\section{Referências}

1. Gussak I, Brugada P, Brugada J, Wright RS, Kopecky SL, Chaitman BR, et al. Idiopathic short QT interval: a new clinical syndrome? Cardiology. 2000;94(2):99-102.

2. Gray B, Ackerman MJ, Semsarian C, Behr ER. Evaluation After Sudden Death in the Young: A Global Approach. Circ Arrhythm Electrophysiol. 2019 Aug;12(8):e007453
3. Gollob MH, Redpath CJ, Roberts JD. The short QT syndrome: proposed diagnostic criteria. J Am Coll Cardiol. 2011 Feb 15;57(7):802-12.

4. Priori SG, Blomström-Lundqvist C, Mazzanti A, Blom N, Borggrefe M, Camm J, et al. 2015 ESC Guidelines for the management of patients with ventricular arrhythmias and the prevention of sudden cardiac Death. The Task Force for the Management of Patients with Ventricular Arrhythmias and the Prevention 


\section{Relato de Caso}

of Sudden Cardiac Death of the European Society of Cardiology. Eur Heart J. 2015 Nov 1;36(41):2793-867.

5. Giudicessi JR, Ackerman MJ. Determinants of incomplete penetrance and variable expressivity in heritable cardiac arrhythmia syndromes. Transl Res. $2013 ; 161(1): 1-14$.

6. Tse G, Chan YW, Keung W, Yan BP. Electrophysiological mechanisms of long and short QT syndromes. Int J Cardiol Heart Vasc. 2016 Nov 26;14:8-13.

7. Yamagata K, Horie M, Aiba T, Ogawa S, Aizawa Y, Ohe T, et al. Genotype-Phenotype Correlation of SCN5A Mutation for the Clinical and Electrocardiographic Characteristics of Probands With Brugada
Syndrome: A Japanese Multicenter Registry. Circulation. 2017 Jun $6 ; 135(23): 2255-70$

8. Giudicessi JR, Ackerman MJ. Genotype-and phenotype-guided management of congenital long QT syndrome. Curr Probl Cardiol. 2013;38(10):417-55.

9. Roston TM, Yuchi Z, Kannankeril PJ, Hathaway J, Vinocur JM, Etheridge SP, et al. The clinical and genetic spectrum of catecholaminergic polymorphic ventricular tachycardia: findings from an international multicentre registry. Europace. 2018;20(3):541-7.

10. Mazzanti A, Kanthan A, Monteforte N, Memmi M, Bloise R, Novelli V, et al. Novel insight into the natural history of short QT syndrome. J Am Coll Cardiol. 2014 Apr 8;63(13):1300-8. 